\title{
THE
}

2008

\section{Quantum Integrability and Nonintegrability in the Spin-boson Model}

Vyacheslav V. Stepanov

University of Rhode Island

Gerhard Müller

University of Rhode Island, gmuller@uri.edu

Joachim Stolze

Follow this and additional works at: https://digitalcommons.uri.edu/phys_facpubs

Terms of Use

All rights reserved under copyright.

\section{Citation/Publisher Attribution}

Stepanov, V. V., Müller, G., \& Stolze, J. (2008). Quantum integrability and nonintegrability in the spin-boson model. Physical Review E, 77(6), 066202, 1-9. doi: 10.1103/PhysRevE.77.066202

Available at: http://dx.doi.org/10.1103/PhysRevE.77.066202

This Article is brought to you for free and open access by the Physics at DigitalCommons@URI. It has been accepted for inclusion in Physics Faculty Publications by an authorized administrator of DigitalCommons@URI. For more information, please contact digitalcommons-group@uri.edu. 


\title{
Quantum integrability and nonintegrability in the spin-boson model
}

\author{
Vyacheslav V. Stepanov, ${ }^{1}$ Gerhard Müller, ${ }^{1}$ and Joachim Stolze ${ }^{2}$ \\ ${ }^{1}$ Department of Physics, University of Rhode Island, Kingston, Rhode Island 02881, USA \\ ${ }^{2}$ Institut für Physik, Technische Universität Dortmund, 44221 Dortmund, Germany
}

(Received 20 February 2008; published 4 June 2008)

\begin{abstract}
We study the spectral properties of a spin-boson Hamiltonian that depends on two continuous parameters $0 \leq \Lambda<\infty$ (interaction strength) and $0 \leq \alpha \leq \pi / 2$ (integrability switch). In the classical limit, this system has two distinct integrable regimes, $\alpha=0$ and $\alpha=\pi / 2$. For each integrable regime we can express the quantum Hamiltonian as a function of two action operators. Their eigenvalues (multiples of $\hbar$ ) are the natural quantum numbers for the complete level spectrum. This functional dependence cannot be extended into the nonintegrable regime $(0<\alpha<\pi / 2)$. Here level crossings are prohibited and the level spectrum is naturally described by a single (energy sorting) quantum number. In consequence, the tracking of individual eigenstates along closed paths through both regimes leads to conflicting assignments of quantum numbers. This effect is a useful and reliable indicator of quantum chaos - a diagnostic tool that is independent of any level-statistical analysis.
\end{abstract}

DOI: 10.1103/PhysRevE.77.066202

PACS number(s): 05.45.Mt

\section{INTRODUCTION}

Classical integrability of a system with two degrees of freedom guarantees that the Hamiltonian can be expressed as a piecewise smooth function of two action coordinates $H\left(p_{1}, q_{1} ; p_{2}, q_{2}\right)=H_{C}\left(J_{1}, J_{2}\right)$. No such functional relation exists if the system is nonintegrable [1-4].

Geometrically speaking, for a parametric system with parameters subject to an integrability condition, there exist complete foliations of invariant tori in phase space for all parameter points in the integrable regime. Throughout the nonintegrable regime the foliation is partially destroyed. Some tori are replaced by chaotic trajectories, cantori, and unstable periodic trajectories. The surviving tori in the nonintegrable regime are no longer dense anywhere in phase space. Whereas each surviving torus can still be characterized by two local action coordinates $J_{1}, J_{2}$ via line integrals $\oint p_{i} d q_{i}$ along pairs of topologically independent closed paths, the functional relation $H_{C}\left(J_{1}, J_{2}\right)$ breaks down at the edge of the integrable regime.

There exists a quantum counterpart to this breakdown, as will be demonstrated. It can be employed to discriminate between regimes of integrability and nonintegrability on purely quantum mechanical grounds. Here we show the workings of this diagnostic tool [5] in the context of the spin-boson model [6-9],

$$
\begin{aligned}
H= & \hbar \omega_{B} a^{\dagger} a+\hbar \omega_{S} S_{z}+\Lambda \cos \alpha\left(S_{+} a+S_{-} a^{\dagger}\right) \\
& +\Lambda \sin \alpha\left(S_{+} a^{\dagger}+S_{-} a\right),
\end{aligned}
$$

one of the simplest nontrivial models describing nonrelativistically the interaction between an atom and a radiation field. This model has also been used to describe the interaction between electronic and vibrational degrees of freedom in molecules and solids. The relation between classical and quantum integrability of Eq. (1) has been the object of previous investigations $[8,10]$.

The Bose operators $a^{\dagger}, a$ in Eq. (1) describe one mode with frequency $\omega_{B}$ of the electromagnetic field. The spin- $\sigma$ operators $S_{ \pm}=S_{x} \pm i S_{y}, S_{z}$ act in the Hilbert space of a $(2 \sigma$ $+1)$-level atom with level spacing $\hbar \omega_{S}$. The coupling be- tween the two degrees of freedom has strength $\Lambda$ and depends on a continuous parameter $\alpha$ that connects two regimes for which this model is integrable in the classical limit. The classical integrability for $\alpha=0$ and $\pi / 2$ is established by a second integral of the motion. The case $\alpha=0$ is known as the rotating wave approximation in quantum optics. Early studies in one or the other classical limit of the spin-boson model revealed chaotic phase space flow turning regular in the rotating wave approximation $[6,7,11,12]$.

In the two-dimensional parameter space spanned by the (polar) coordinates $(\Lambda, \alpha)$, the two integrable regimes are located on two perpendicular straight lines that intersect each other at the point of zero coupling strength. Each quadrant of this parameter plane represents a nonintegrable regime. Henceforth we consider the parameter range $0 \leq \Lambda<\infty, 0$ $\leq \alpha \leq \pi / 2$.

In preparation for our main theme, we first discuss the classical integrability condition of the spin-boson model (Sec. II) and then the classification of its quantum energy levels (Sec. III) and certain quantum invariants (Sec. IV) by distinct sets of quantum numbers in the integrable and nonintegrable regimes. This distinction has a deeper meaning, which we will further discuss in Sec. V, and which we will employ in Sec. VI for the identification of the two regimes in purely quantum mechanical terms.

\section{INTEGRABILITY CONDITION}

In taking the classical limit $\hbar \rightarrow 0, \sigma \rightarrow \infty$ of the spinboson model, we renormalize the coupling constant $\Lambda$ $=(\hbar / 2)^{3 / 2} \bar{\Lambda}$, substitute

$$
a=x \sqrt{M \omega_{B} / 2 \hbar}+i p / \sqrt{2 \hbar M \omega_{B}}
$$

for the boson operators, and, via $\hbar \sqrt{\sigma(\sigma+1)}=s$, convert the spin- $\sigma$ operator into a classical three-component vector of fixed length,

$$
\left(S_{x}, S_{y}, S_{z}\right)=s(\sin \vartheta \cos \varphi, \sin \vartheta \sin \varphi, \cos \vartheta) .
$$

The spin-boson Hamiltonian (1) thus turns into the energy function of two linear one-degree-of-freedom systems-a 
harmonic oscillator and a classical spin in a constant magnetic field-with a nonlinear coupling,

$$
\begin{aligned}
H= & \frac{p^{2}}{2 M}+\frac{1}{2} M \omega_{B}^{2} x^{2}+\omega_{S} S_{z}+\frac{1}{2} \bar{\Lambda} \cos \alpha\left(\sqrt{M \omega_{B}} x S_{x}\right. \\
& \left.-\frac{1}{\sqrt{M \omega_{B}}} p S_{y}\right)+\frac{1}{2} \bar{\Lambda} \sin \alpha\left(\sqrt{M \omega_{B}} x S_{x}+\frac{1}{\sqrt{M \omega_{B}}} p S_{y}\right) .
\end{aligned}
$$

A set of canonical coordinates is $(p, x ; s \cos \vartheta, \varphi)$. The equations of motion for the physical variables $\left(x, p, S_{x}, S_{y}, S_{z}\right)$ inferred from Eq. (4) via $d x / d t=\partial H / \partial p, d p / d t=-\partial H / \partial x$, and $d \mathbf{S} / d t=-\mathbf{S} \times \partial H / \partial \mathbf{S}$ read

$$
\begin{gathered}
\dot{x}=\frac{p}{M}+\frac{\bar{\Lambda}}{2 \sqrt{M \omega_{B}}} S_{y}(\sin \alpha-\cos \alpha), \\
\dot{p}=-M \omega_{B}^{2} x-\frac{\bar{\Lambda} \sqrt{M \omega_{B}}}{2} S_{x}(\cos \alpha+\sin \alpha), \\
\dot{S}_{x}=-\omega_{S} S_{y}-\frac{\bar{\Lambda} p}{2 \sqrt{M \omega_{B}}} S_{z}(\cos \alpha-\sin \alpha), \\
\dot{S}_{y}=\omega_{S} S_{x}-\frac{\bar{\Lambda} x \sqrt{M \omega_{B}}}{2} S_{z}(\cos \alpha+\sin \alpha), \\
\dot{S}_{z}=\frac{\bar{\Lambda} x \sqrt{M \omega_{B}}}{2} S_{y}(\cos \alpha+\sin \alpha)+\frac{\bar{\Lambda} p}{2 \sqrt{M \omega_{B}}} S_{x}(\cos \alpha-\sin \alpha)
\end{gathered}
$$

The phase flow generated by these equations is, in general, chaotic. Chaos gives way to a fully intact torus structure at $\alpha=0, \pi / 2$. The integrability of these cases is established by the fact that one or the other of the two functions

$$
\begin{gathered}
I=\frac{p^{2}}{2 M \omega_{B}}+\frac{1}{2} M \omega_{B} x^{2}+S_{z}, \\
K=\frac{p^{2}}{2 M \omega_{B}}+\frac{1}{2} M \omega_{B} x^{2}-S_{z},
\end{gathered}
$$

whose time evolution is determined by [13]

$$
\begin{gathered}
\dot{I}=\{H, I\}=\bar{\Lambda} \sin \alpha\left(\frac{p S_{x}}{\sqrt{M \omega_{B}}}-\sqrt{M \omega_{B}} x S_{y}\right), \\
\dot{K}=\{H, K\}=\bar{\Lambda} \cos \alpha\left(\frac{p S_{x}}{\sqrt{M \omega_{B}}}+\sqrt{M \omega_{B}} x S_{y}\right),
\end{gathered}
$$

becomes a second integral of the motion. The case $\alpha=0$ is known as the Jaynes-Cummings model [14]. The impact of the classical integrability conditions on the quantum system is the main theme of this study.

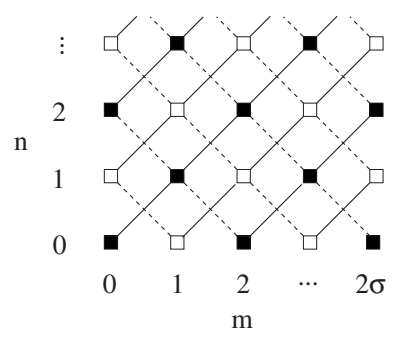

FIG. 1. Basis vectors $|m, n\rangle$ with positive (full squares) and negative parity (open squares) as coupled by matrix elements of operators $S_{+} a, S_{-} a^{\dagger}$ (solid lines) and $S_{+} a^{\dagger}, S_{-} a$ (dashed lines) of Hamiltonian (1).

\section{ENERGY LEVELS}

For the analytic or numerical solution of the spin-boson model (1), it is convenient to use the product vectors of the noninteracting system, $|m, n\rangle, \quad m=0,1,2, \ldots, 2 \sigma, n$ $=0,1,2, \ldots$, as a basis. The relevant operators act on this basis as follows:

$$
\begin{gathered}
\left(\sigma-S_{z}\right)|m, n\rangle=m|m, n\rangle, \\
S_{+}|m, n\rangle=\sqrt{m(2 \sigma-m+1)}|m-1, n\rangle, \\
S_{-}|m, n\rangle=\sqrt{(2 \sigma-m)(m+1)}|m+1, n\rangle, \\
a^{\dagger}|m, n\rangle=\sqrt{n+1}|m, n+1\rangle, \\
a|m, n\rangle=\sqrt{n}|m, n-1\rangle .
\end{gathered}
$$

The Hamiltonian matrix can thus be assembled from the diagonal elements

$$
\left\langle m, n\left|S_{z}\right| m, n\right\rangle=\sigma-m, \quad\left\langle m, n\left|a^{\dagger} a\right| m, n\right\rangle=n,
$$

and from the off-diagonal elements

$$
\begin{gathered}
\left\langle m, n\left|S_{+} a\right| m+1, n+1\right\rangle=\sqrt{(2 \sigma-m)(m+1)(n+1)}, \\
\left\langle m, n\left|S_{-} a^{\dagger}\right| m-1, n-1\right\rangle=\sqrt{(2 \sigma+1-m) m n}, \\
\left\langle m, n\left|S_{+} a^{\dagger}\right| m+1, n-1\right\rangle=\sqrt{(2 \sigma-m)(m+1) n}, \\
\left\langle m, n\left|S_{-} a\right| m-1, n+1\right\rangle=\sqrt{(2 \sigma+1-m) m(n+1)} .
\end{gathered}
$$

The structure of this matrix is illustrated in Fig. 1. The solid lines represent matrix elements generated by the first interaction term in Eq. (1), and the dashed lines represent matrix elements which arise in the second interaction term. The two-sublattice structure is a reflection of parity conservation. The parity operator

$$
P=(-1)^{a^{\dagger} a+\sigma-S_{z}}
$$

commutes with $H$ for arbitrary $\Lambda, \alpha$. It divides the eigenstates into two symmetry classes. States with $P=+1(P$ $=-1)$ involve basis vectors with even $m+n$ (odd $m+n)$ only. 
If $\alpha=0$ only the solid bonds are present and if $\alpha=\pi / 2$ only the dashed bonds. In either case the Hamiltonian matrix is reduced to invariant blocks of size $2 \sigma+1$. If $0<\alpha<\pi / 2$ we must deal with an infinite matrix. In this study we set $\omega_{S}=\omega_{B} \equiv \omega$ except where indicated otherwise. In the following we analyze the level spectrum for various cases at $\alpha$ $=0, \pi / 2$.

The integrable spin-boson model with $\sigma=\frac{1}{2}$ involves only $2 \times 2$ matrices. If $\alpha=0$, the eigenvectors happen to be independent of the interaction strength,

$$
\begin{gathered}
\left|\psi_{1,0}\right\rangle=|1,0\rangle \\
\left|\psi_{1, n}\right\rangle=\frac{1}{\sqrt{2}}\{|1, n\rangle+|0, n-1\rangle\}, \quad n=1,2, \ldots, \\
\left|\psi_{0, n}\right\rangle=\frac{1}{\sqrt{2}}\{|1, n+1\rangle-|0, n\rangle\}, \quad n=0,1, \ldots,
\end{gathered}
$$

and the energy eigenvalues (for $n=0,1,2, \ldots$ ) are

$$
\begin{gathered}
E_{1, n}=\hbar \omega(n-1 / 2)+\Lambda \sqrt{n}, \\
E_{0, n}=\hbar \omega(n+1 / 2)-\Lambda \sqrt{n+1} .
\end{gathered}
$$

If $\alpha=\pi / 2$ the eigenvectors do depend on $\Lambda$ :

$$
\begin{gathered}
\left|\psi_{1,0}\right\rangle=|0,0\rangle, \\
\left|\psi_{1, n}\right\rangle=a_{0, n}|0, n\rangle+b_{0, n}|1, n-1\rangle, \quad n=1,2, \ldots, \\
\left|\psi_{0, n}\right\rangle=a_{1, n}|0, n+1\rangle-b_{1, n+1}|1, n\rangle, \quad n=0,1, \ldots,
\end{gathered}
$$

with

$$
\begin{aligned}
& a_{0, n}=\frac{\sqrt{\lambda_{n}-1}}{\sqrt{2\left(\lambda_{1}-\sqrt{\lambda_{n}}\right)}}, \quad a_{1, n-1}=\frac{\sqrt{\lambda_{n}-1}}{\sqrt{2\left(\lambda_{1}+\sqrt{\lambda_{n}}\right)}}, \\
& b_{0, n}=\frac{\sqrt{\lambda_{n}}-1}{\sqrt{2\left(\lambda_{1}-\sqrt{\lambda_{n}}\right)}}, \quad b_{1, n-1}=\frac{\sqrt{\lambda_{n}}+1}{\sqrt{2\left(\lambda_{1}+\sqrt{\lambda_{n}}\right)}},
\end{aligned}
$$

where $\lambda \doteq(\Lambda / \hbar \omega)^{2}$ and $\lambda_{n} \doteq 1+n \lambda$. The associated energy eigenvalues (for $n=0,1,2, \ldots$ ) are

$$
\begin{gathered}
E_{1, n}=\hbar \omega(n-1 / 2)+\hbar \omega \sqrt{\lambda_{n}}, \\
E_{0, n}=\hbar \omega(n+1 / 2)-\hbar \omega \sqrt{\lambda_{n+1}} .
\end{gathered}
$$

Additional results (for $\sigma>\frac{1}{2}$ ) can be found in Appendix A. In all cases pertaining to the integrable regimes $\alpha=0$ or $\pi / 2$ the energy levels are naturally labeled by the two quantum numbers $m, n$. The parity becomes $P=(-1)^{m+n}$. In the nonintegrable regime $0<\alpha<\pi / 2$, by contrast, the numerical analysis suggests that no two energy levels of the same parity become degenerate or undergo any crossing upon variation of the parameters $\Lambda, \alpha[15]$. All levels of a given parity can thus be unambiguously labeled by a single (energy sorting) quantum number $k$.

\section{QUANTUM INVARIANTS}

The quantum counterparts to the two analytic invariants (6a) and (6b) are the operators

$$
I=\hbar\left(a^{\dagger} a+S_{z}\right), \quad K=\hbar\left(a^{\dagger} a-S_{z}\right),
$$

which indeed commute with Eq. (1) under exactly the same conditions as in the classical limit. We have

$$
\begin{gathered}
{[H, I]=2 \Lambda \sin \alpha\left(S_{-} a-S_{+} a^{\dagger}\right),} \\
{[H, K]=2 \Lambda \cos \alpha\left(S_{+} a-S_{-} a^{\dagger}\right) .}
\end{gathered}
$$

However, quantum integrability cannot be inferred from quantum invariants as simply as classical integrability can be inferred from integrals of the motion (analytic invariants). Commuting operators can always be constructed irrespective of whether the model is (classically) integrable or not $[16,17]$. The parity operator (10), for example, which can be expressed as a function of either invariant $I$ or $K$,

$$
P=e^{i \pi(I / \hbar-\sigma)}=e^{i \pi(K / \hbar+\sigma)},
$$

commutes with $H$ for arbitrary $\alpha$. More generally, any operator $A$ that is not already an invariant, $[H, A] \neq 0$, can be turned into an invariant via time averaging $[18,19]$. Consider the matrix elements of $A(t)$ in the energy representation, $\left\langle\nu|A| \nu^{\prime}\right\rangle e^{i\left(E_{\nu^{\prime}}-E_{\nu^{\prime}}\right) t / \hbar}$. Barring degeneracies, the time average removes all off-diagonal elements:

$$
\overline{\left\langle\nu|A(t)| \nu^{\prime}\right\rangle} \doteq \lim _{T \rightarrow \infty} \frac{1}{T} \int_{0}^{\infty} d t\left\langle\nu|A(t)| \nu^{\prime}\right\rangle=\left\langle\nu|A| \nu^{\prime}\right\rangle \delta_{\nu \nu^{\prime}} .
$$

The resulting operator $I_{A}$ with (diagonal) elements $\langle\nu|A| \nu\rangle$ thus commutes with $H$ by construction.

The fact is that in the classical limit neither the parity operator nor any of the artificially constructed quantum invariants will turn into analytic invariants (integrals of the motion) if the phase flow is chaotic. Such quantum invariants either lose their meaning altogether or turn into nonanalytic invariants $[19,20]$.

The distinctive attributes of quantum invariants in the integrable and nonintegrable regimes of a quantum system are subtle but not ambiguous. Here we use

$$
I_{A}=\langle A\rangle, \quad A=a^{\dagger}\left(S_{-}+S_{+}\right) .
$$

For $\sigma=\frac{1}{2}$, its eigenvalues at $\alpha=0$ can be calculated from the eigenvectors (11a)-(11c),

$$
\langle A\rangle_{1, n}=\frac{1}{2} \sqrt{n}, \quad\langle A\rangle_{0, n}=-\frac{1}{2} \sqrt{n+1},
$$

and its eigenvalues at $\alpha=\pi / 2$ from the eigenvectors (13a) $-(13 c)$,

$$
\langle A\rangle_{1, n}=\frac{\left(\lambda_{n}-1\right)\left(\sqrt{\lambda_{n}}-1\right)}{2\left(\lambda_{n}-\sqrt{\lambda_{n}}\right)},
$$




$$
\langle A\rangle_{0, n}=-\frac{\left(\lambda_{n+1}-1\right)\left(\sqrt{\lambda_{n+1}}+1\right)}{2\left(\lambda_{n+1}+\sqrt{\lambda_{n+1}}\right)} .
$$

Appendix A contains results for $\sigma>\frac{1}{2}$. Numerical results of $\langle A\rangle_{k}$ for $0<\alpha<\pi / 2$ of systems with $\sigma=\frac{1}{2}, 1, \frac{3}{2}$ were reported previously [9]. The patterns of points $\left(E_{m, n},\langle A\rangle_{m, n}\right)$ for integrable cases were found to be strikingly different from the pattern of points $\left(E_{k},\langle A\rangle_{k}\right)$ for nonintegrable cases. Here this difference will be used as a demarcation tool for regimes of integrability and nonintegrability.

\section{QUANTUM ACTIONS}

One signature of integrability in a quantum system with two degrees of freedom is that the Hamiltonian can be expressed as a function of two action operators $J_{1}, J_{2}$, i.e., of two quantum invariants whose spectra consist of equidistant levels [1-3]. In the absence of the spin-boson interaction $(\Lambda=0)$, the two action operators are

$$
J_{1}=\hbar\left(\sigma-S_{z}\right), \quad J_{2}=\hbar a^{\dagger} a,
$$

with integer eigenvalues (in units of $\hbar$ )

$$
\begin{gathered}
J_{1}=m \hbar, \quad m=0,1, \ldots, 2 \sigma, \\
J_{2}=n \hbar, \quad n=0,1, \ldots,
\end{gathered}
$$

as in Eq. (9). The Hamiltonian $H_{0}=\hbar \omega_{B} a^{\dagger} a+\hbar \omega_{S} S_{z}$ and the two quantum invariants (15) are expressible as linear combinations of $J_{1}, J_{2}$.

Classically, the contribution of each degree of freedom to $H_{0}=p^{2} / 2 M+\frac{1}{2} M \omega_{B}^{2} x^{2}+\omega_{S} S_{z}$ is transformed into a function of one action coordinate by a separate canonical transformation: $\left(S_{z}, \varphi\right) \rightarrow\left(J_{1}, \theta_{1}\right)$ with $S_{z}=s-J_{1}, \varphi=-\theta_{1}$, and $(p, x)$ $\rightarrow\left(J_{2}, \theta_{2}\right)$ with $p=\sqrt{2 J_{2} M \omega_{B}} \cos \theta_{2}, \quad x=\sqrt{2 J_{2} / M \omega_{B}} \sin \theta_{2}$. The transformed Hamiltonian and the two classical invariants (6a) and (6b) are linear functions of $J_{1}, J_{2}$ just as in quantum mechanics. The exact quantum spectra of $H_{0}, I, K$ can then be recovered exactly via semiclassical quantization, i.e., by substituting the actions quantized according to Eqs. (22a) and (22b) into the classical Hamiltonian.

Classically, the interaction renders the equations of motion, Eqs. (5a)-(5e), nonlinear. However, the effects of anharmonicity in the time evolution depend sensitively on whether integrability is sustained or destroyed by the interaction. Integrability for $\alpha=0, \pi / 2$ dictates that the phase flow is exclusively toroidal. For $0<\alpha<\pi / 2$ chaotic phase flow is omnipresent, albeit constrained by surviving tori.

Quantum mechanically, the interaction distorts the eigenvalue spectrum and modifies the selection rules of transition rates. Quantum properties that are as sensitive to the integrability status as their classical counterparts do exist and have previously been explored in the context of a different model system [21-23].

These properties are directly related to the existence of action operators as constituent elements of the Hamiltonian. For the noninteracting system we have Eq. (21). In the interacting cases, the existence of action operators can again be demonstrated directly for $\alpha=0, \pi / 2$, and their nonexistence for $0<\alpha<\pi / 2$ can be demonstrated indirectly.
The unitary transformation which diagonalizes the Hamiltonian (1) for $\sigma=\frac{1}{2}$ and $\alpha=0$, expressed in terms of spin and boson operators, reads

$$
U_{A}=P_{0}^{A}+\frac{1}{\sqrt{2}}\left(-2 S_{z}+\frac{1}{\sqrt{a^{\dagger} a}} a^{\dagger} S_{-}+a S_{+} \frac{1}{\sqrt{a^{\dagger} a}} Q_{1}^{A}\right),
$$

where $P_{0}^{A}=|1,0\rangle\left\langle 1,0\left|, Q_{1}^{A}=1-\right| 0,0\right\rangle\langle 0,0|-| 1,0\rangle\langle 1,0|$. The operators

$$
T_{z}=U_{A} S_{z} U_{A}^{-1}=P_{0}^{A} S_{z}-\frac{1}{2} G_{1}^{A},
$$

$$
b^{\dagger} b=U_{A} a^{\dagger} a U_{A}^{-1}=a^{\dagger} a-S_{z} P_{0}^{A}+\frac{1}{2} G_{1}^{A},
$$

with

$$
G_{1}^{A}=a S_{+} \frac{1}{\sqrt{a^{\dagger} a}} Q_{1}^{A}+\frac{1}{\sqrt{a^{\dagger} a}} a^{\dagger} \hat{S}_{-}
$$

are diagonal in the energy representation:

$$
\begin{gathered}
T_{z}\left|\psi_{m, n}\right\rangle=(\sigma-m)\left|\psi_{m, n}\right\rangle, \\
b^{\dagger} b\left|\psi_{m, n}\right\rangle=n\left|\psi_{m, n}\right\rangle .
\end{gathered}
$$

Hence the quantum actions with eigenvalues (22a) and (22b) are

$$
J_{1}=\hbar\left(\sigma-T_{z}\right), \quad J_{2}=\hbar b^{\dagger} b .
$$

Applying $U_{A}$ to the Hamiltonian yields

$$
\begin{aligned}
U_{A} H U_{A}^{-1}= & \hbar \omega\left(b^{\dagger} b+T_{z}\right)+\Lambda\left(\frac{1-2 T_{z}}{2} \sqrt{b^{\dagger} b}\right. \\
& \left.-\frac{1+2 T_{z}}{2} \sqrt{b^{\dagger} b+1}\right),
\end{aligned}
$$

which, together with Eq. (26), describes the functional relation between $H$ and $J_{1}, J_{2}$.

The same method also produces the quantum actions for the integrable case $s=\frac{1}{2}, \alpha=\pi / 2$. Here the block-diagonal unitary transformation $U_{B}$ to be used can also be expressed in terms of spin and boson operators but has a more complicated structure than $U_{A}$. The operators

$$
\begin{gathered}
T_{z}=U_{B} S_{z} U_{B}^{-1}=\frac{1}{2} P_{0}^{B}+\left(G_{B}\right) Q_{0}^{B}, \\
b^{\dagger} b=U_{B} a^{\dagger} a U_{B}=\left(a^{\dagger} a-S_{z}+G_{B}\right) Q_{0}^{B},
\end{gathered}
$$

with $G_{B}=G_{1}^{B}+G_{2}^{B}+G_{3}^{B}$,

$$
\begin{gathered}
G_{1}^{B}=\frac{1}{\sqrt{16\left(1+\lambda a^{\dagger} a\right)}}-\frac{1}{\sqrt{16\left[1+\lambda\left(a^{\dagger} a+1\right)\right]}}, \\
G_{2}^{B}=\frac{S_{z}}{\sqrt{4\left(1+\lambda a^{\dagger} a\right)}}+\frac{S_{z}}{\sqrt{4\left[1+\lambda\left(a^{\dagger} a+1\right)\right]}},
\end{gathered}
$$




$$
G_{3}^{B}=\frac{1+2 S_{z}}{4 \sqrt{64 \lambda+a^{\dagger} a}} a^{\dagger} S_{+}+a S_{-} \frac{1+2 S_{z}}{4 \sqrt{64 \lambda+a^{\dagger} a}},
$$

and $P_{0}^{B}=|0,0\rangle\left\langle 0,0\left|, Q_{0}^{B}=1-\right| 0,0\right\rangle\langle 0,0|$, again satisfy Eqs. (25a) and (25b) and are related to quantum actions via Eq. (26). The functional dependence of the transformed Hamiltonian on the actions is different from Eq. (27):

$$
\begin{aligned}
U_{B} H U_{B}^{-1}= & \hbar \omega\left(b^{\dagger} b-T_{z}\right)+\frac{1+2 T_{z}}{2} \sqrt{1+\lambda b^{\dagger} b} \\
& -\frac{1-2 T_{z}}{2} \sqrt{1+\lambda\left(b^{\dagger} b+1\right)} .
\end{aligned}
$$

$U_{A}$ is the special case for $\sigma=\frac{1}{2}$ of a unitary transformation $U_{1}(\sigma, \Lambda)$ that diagonalizes Eq. (1) at $\alpha=0$ for arbitrary values of $\sigma$. Likewise, $U_{B}$ is the special case for $\sigma=\frac{1}{2}$ of a unitary transformation $U_{2}(\sigma, \Lambda)$ that diagonalizes Eq. (1) at $\alpha=\pi / 2$ for arbitrary $\sigma$ (see Appendix A for additional results).

The end product of these unitary transformations are two functions $\bar{H}_{Q}^{(1)}\left(T_{z}, b^{\dagger} b ; \Lambda\right)=H_{Q}^{(1)}\left(J_{1}, J_{2} ; \Lambda\right)$ and $\bar{H}_{Q}^{(2)}\left(T_{z}, b^{\dagger} b ; \Lambda\right)=H_{Q}^{(2)}\left(J_{1}, J_{2} ; \Lambda\right)$, which express the functional dependence of the Hamiltonian on action operators in the two integrable regimes $\alpha=0$ and $\pi / 2$, respectively. The leading terms of an asymptotic expansion at high boson occupancy and unrestricted spin state of these functions are

$$
\bar{H}_{Q}=\hbar \omega\left(b^{\dagger} b \pm T_{z}\right)+\Lambda \sqrt{b^{\dagger} b}+O(1),
$$

where the operators $T_{z}, b^{\dagger}, b$ again satisfy Eqs. (25a) and (25b) and the upper (lower) sign pertains to $\alpha=0(\alpha=\pi / 2)$.

We expect a semiclassical regime to exist at large spin and/or boson quantum numbers where the functions $H_{Q}^{(1)}\left(J_{1}, J_{2} ; \Lambda\right)$ and $H_{Q}^{(2)}\left(J_{1}, J_{2} ; \Lambda\right)$ connect with functions $H_{C}^{(1)}\left(J_{1}, J_{2} ; \Lambda\right)$ and $H_{C}^{(2)}\left(J_{1}, J_{2} ; \Lambda\right)$ of classical actions. However, the identification of the semiclassical regime requires a complete solution of the classical equations of motion (5a)-(5e), a task still outstanding.

The connections between the quantum and classical functional dependences of Hamiltonian on actions was investigated in a previous study for an integrable two-spin model and for the (integrable) circular billiard model [23]. There we found subtle quantum effects that restrict the range of the semiclassical regime in unexpected ways. That may also be the case in the spin-boson model. However, the point we wish to emphasize in this study is a different one.

\section{TRACKING EIGENSTATES}

The goal is to demonstrate that the functions $H_{Q}^{(1)}\left(J_{1}, J_{2} ; \Lambda\right)$ and $H_{Q}^{(2)}\left(J_{1}, J_{2} ; \Lambda\right)$ cannot be extended in any consistent way into the region of nonintegrability in the $(\Lambda, \alpha)$ plane. The functions $H_{Q}^{(1)}$ and $H_{Q}^{(2)}$ make it possible to label all eigenstates of Eq. (1) by the two action quantum numbers $m, n$ as defined in Eqs. (22a) and (22b) and to track them with no ambiguity through each one of the two integrable regimes $\alpha=0$ and $\pi / 2$. The nonextendability of the two functions $H_{Q}^{(1)}$ and $H_{Q}^{(2)}$ into a function $H_{Q}\left(J_{1}, J_{2} ; \Lambda, \alpha\right)$ translates into the impossibility of consistently assigning action quantum numbers $m, n$ to the eigenstates in the entire parameter range $0 \leq \Lambda<\infty, 0 \leq \alpha \leq \pi / 2$.

One way of keeping track of eigenstates $|\nu\rangle$ of Eq. (1) is to determine how the eigenvalues of quantum invariants vary along some path in the $(\Lambda, \alpha)$ plane. For the purpose of this demonstration, we focus on the eigenvalues $\langle H\rangle_{\nu}=E_{\nu}$ of the Hamiltonian (1) with spin quantum number $\sigma=\frac{1}{2}$ and the eigenvalues $\langle A\rangle_{\nu}$ of the quantum invariant $I_{A}$ as defined in Eq. (18).

Within each of the two integrable regimes, both sets of eigenvalues have an explicitly known (discrete) dependence on the action quantum numbers $\nu=(m, n)$ and an explicitly known (continuous) dependence on the interaction strength $\Lambda$. The functional relations are stated in Eqs. (12a), (12b), and (19) for $\alpha=0$ and Eqs. (14a), (14b), (20a), and (20b) for $\alpha=\pi / 2$.

\section{A. Level crossings}

In Figs. 2(a) and 2(b) we have plotted one quantum invariant versus the other for all states with positive parity up to a certain energy. In both panels we observe two vertically displaced rows of states. States in the top and bottom rows have action quantum numbers $(1, n)$ and $(0, n)$, respectively.

The observed arrangement of states is due to the fact that $\langle A\rangle_{m, n} \sim \sqrt{n}$ but $E_{m, n} \sim n$ in leading order. Notice that the spacings between successive energy levels in each row vary slowly, and at different rates in the top and bottom rows. To enhance the visibility of this effect we have connected successive energy levels in each panel by dashed lines. The spacings are somewhat larger in the top row compared to the bottom row, causing instances in both panels where two consecutive states of the bottom row fit into the space between two states of the top row. These instances where the alternating (top-bottom) sequence is broken mark locations where energy levels from opposite rows can fall arbitrarily close to each other.

When we increase the interaction strength $\Lambda$ gradually, the states in the top row of Fig. 2(a) move toward the right and the states in the bottom row toward the left. The same observation can be made in Fig. 2(b). Here the shift also contains a small vertical component. We have singled out one pair of nearly degenerate states in Fig. 2(a) and another pair in Fig. 2(b). Each pair is marked by full circles. In Figs. 3(a) and 3(b) we have plotted the traces of these states in the plane of invariants as the interaction strength is increased by a certain amount.

The gradual change of $\Lambda$ causes a cascade of level crossings between states from opposite rows. For the two pairs of tagged states, the crossings occur at the point marked by an asterisk on their traces. States from opposite rows undergo level crossings even though they have the same parity. What matters are the functional relations $H_{Q}^{(1)}$ and $H_{Q}^{(2)}$ established previously. They remove any possible cause for level collisions (avoided crossings) between states from opposite rows as they move (energetically) in opposite directions when $\Lambda$ is increased.

\section{B. Level collisons}

A very different scenario unfurls when we plot the two quantum invariants for a nonintegrable case. What happens 

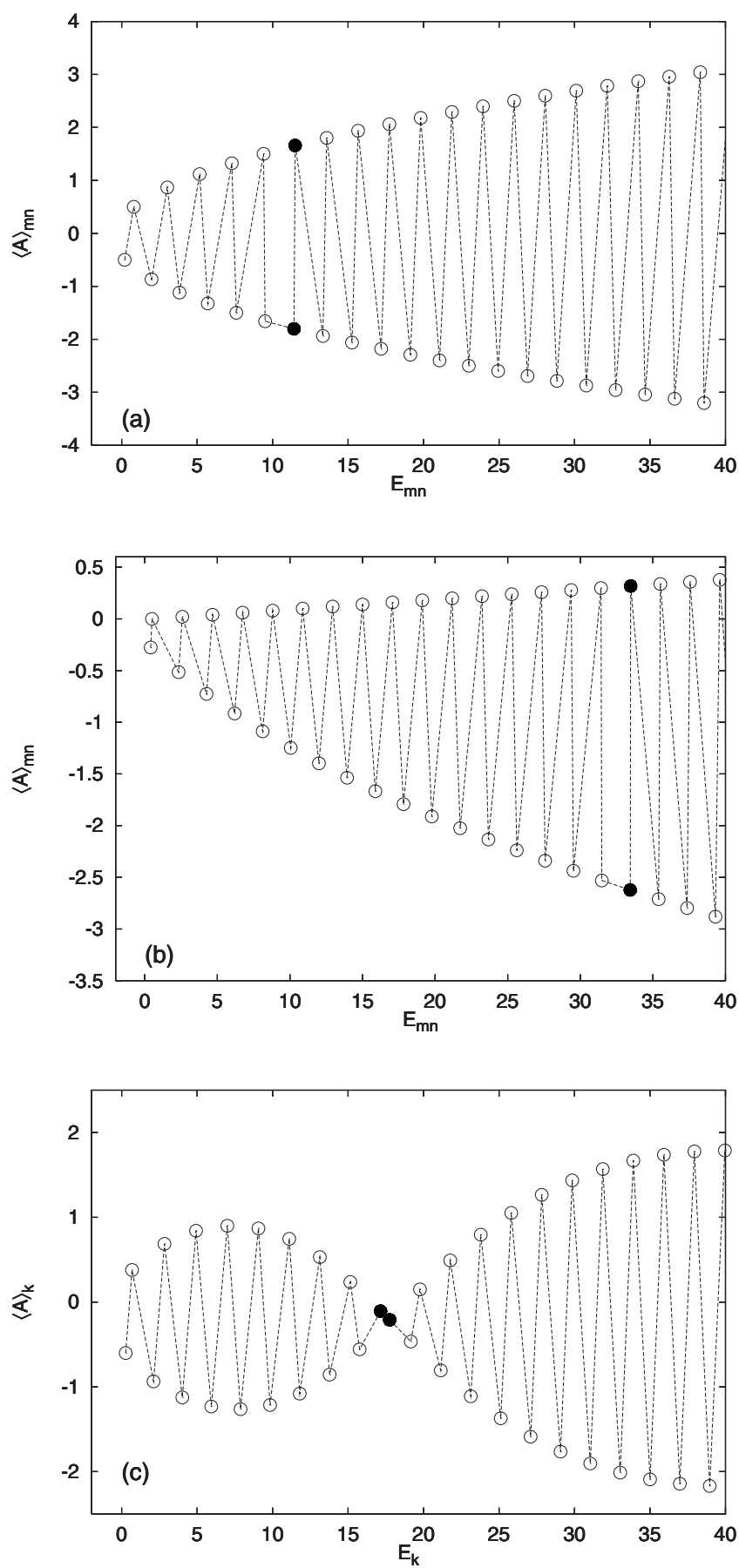

FIG. 2. Quantum invariant $\langle A\rangle_{\nu}=\left\langle\nu\left|a^{\dagger}\left(S_{-}+S_{+}\right)\right| \nu\right\rangle$ versus quantum invariant $E_{\nu}=\langle\nu|H| \nu\rangle$ over some energy range for the eigenstates $|\nu\rangle$ with parity $P=+1$ of the spin-boson model (1) with $\sigma$ $=1 / 2, \hbar \omega=1, \lambda \doteq(\Lambda / \hbar \omega)^{2}=0.09$, and $\alpha=$ (a) 0 , (b) $\pi / 2$, and (c) $\pi / 4$. In the integrable regimes we use the action quantum numbers $\nu=(m, n)$ and in the nonintegrable regime we use the energy sorting quantum number $\nu=k$. One pair of states in each panel (full circles) is tagged for further use in Fig. 3.

when we change the integrability parameter from $\alpha=0$ [Fig. 2(a)] or from $\alpha=\pi / 2$ [Fig. 2(b)] to $\alpha=\pi / 4$ is illustrated in Fig. 2(c). Here the states that used to exist in different worlds (top row with action quantum number $m=1$ and bottom row with $m=0$ ) now suddenly get into each other's way. Since
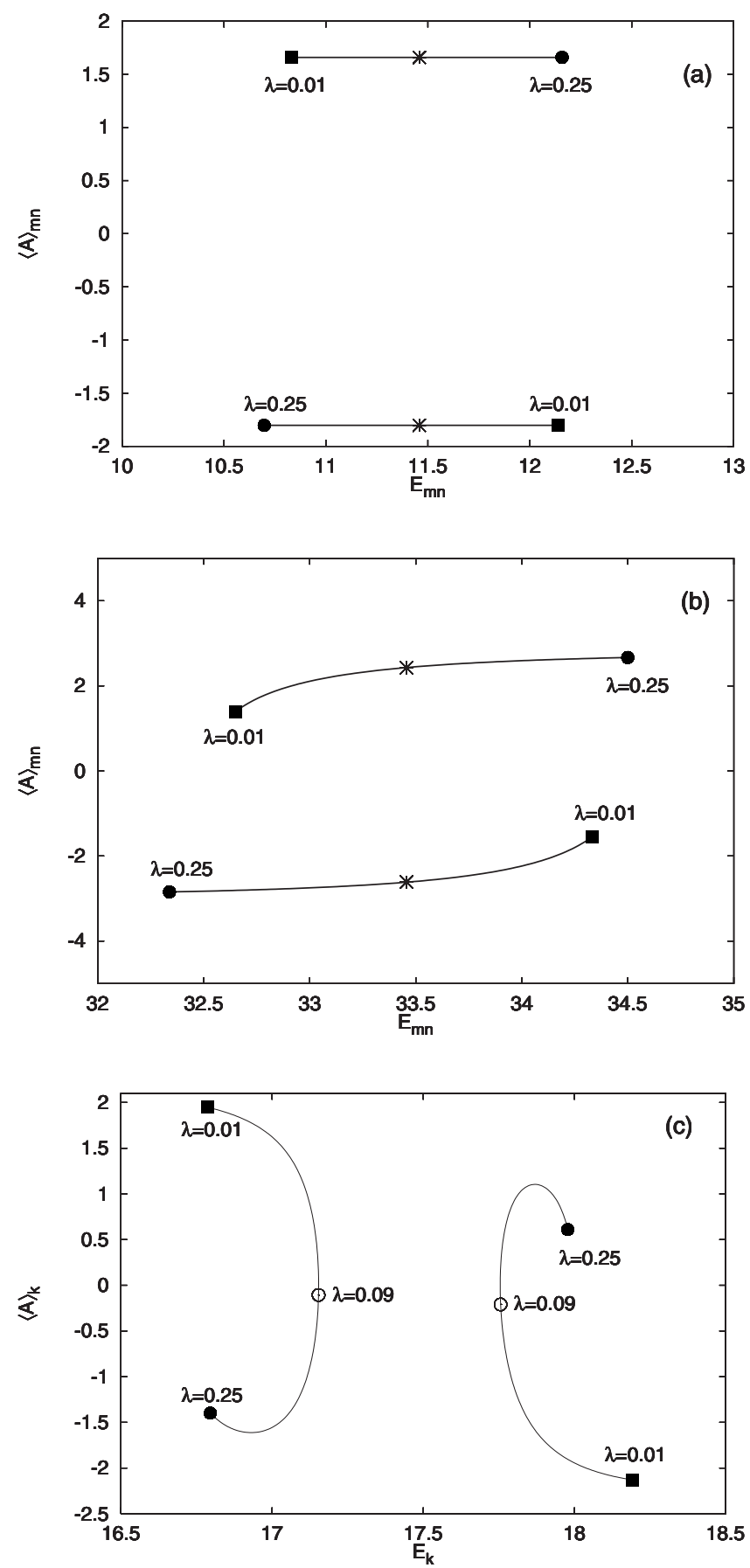

FIG. 3. Trace of one pair of eigenstates $|\nu\rangle$ with parity $P=+1$ (identified by full circles in Fig. 2) in the plane of quantum invariants $\left(E_{\nu},\langle A\rangle_{\nu}\right)$ as the interaction parameter $\lambda$ is increased a specified amount at constant value $\alpha=$ (a) 0 , (b) $\pi / 2$, and (c) $\pi / 4$ of the integrability parameter. In the integrable regimes we use $\nu=(m, n)$ and in the nonintegrable regime $\nu=k$.

they are prohibited from undergoing any level crossings, it is now appropriate to label them by the energy sorting quantum number $k$.

In those parts of the spectrum where the energy level spacings are large, the loss of integrability has no visible effect on the quantum invariants. That is the case near the left and right border areas of Fig. 2(c). Here the two rows of 
states remain largely intact. However, near the center of the panel, where small energy level spacings occur, the eigenvectors of nearly degenerate levels affect each other strongly. The most conspicuous effect is a strong vertical displacement of the two states from the row positions toward each other. Less conspicuous in Fig. 2(c) but of even greater importance is the small horizontal displacement of the two nearly degenerate states away from each other. The effect of nonintegrability is that energy levels exert a short-distance repulsion on each other. At the same time, expectation values in general and the quantum invariant $\langle A\rangle_{k}$ in particular tend to become less differentiated than they were in the integrable case.

When we again increase the interaction strength $\Lambda$, now at fixed $\alpha=\pi / 4$ in the nonintegrable regime, we find that no levels with equal parity ever undergo a crossing. As in the integrable cases, the states with $\langle A\rangle_{k}>0$ have a tendency to move toward the right and the states with $\langle A\rangle_{k}<0$ toward the left.

Inevitably, these trends put states on opposite sides of $\langle A\rangle_{k}=0$ on a collision course. When two such states approach one another, the state starting out with $\langle A\rangle_{k}>0$ swings down as it moves to the right and the state with $\langle A\rangle_{k}<0$ swings up as it moves to the left. The two states reach their closest energetic approach when their vertical positions are about the same. After that, the state coming from below continues its upswing, but now it is moving to the right to join the rightmoving upper row of states. Meanwhile, the state coming from above continues its downswing to join the left-moving lower row of states. One such level collision, between the tagged states in Fig. 2(c), is shown in Fig. 3(c).

\section{Quantum numbers in conflict}

Looking at the spectrum of the spin-boson model (1) in the plane of invariants $\left(E_{\nu},\langle A\rangle_{\nu}\right)$ as the interaction strength $\Lambda$ increases gradually, reveals strikingly different patterns of coordinated motion of all states with given parity, depending on whether the parameter $\alpha$ is set to an integrable regime $(\alpha=0, \pi / 2)$ or fixed within the nonintegrable regime $(0<\alpha$ $<\pi / 2)$.

For $\alpha=0$ or $\pi / 2$ [panels (a) and (b), respectively, of Figs. 2 and 3], the two rows of states march past each other in an orderly fashion, undergoing a sequence of level crossings in complete oblivion of each other's presence. For $\alpha=\pi / 4$ [panel (c) of Figs. 2 and 3], on the other hand, all states are part of a coordinated clockwise looping motion. While every individual state maintains the same position in the level sequence, the wave-shaped top row of states has the appearance of moving steadily to the right and the bottom row to the left. The path of an individual state in the plane of invariants is not unlike that of an $\mathrm{H}_{2} \mathrm{O}$ molecule in a traveling surface water wave.

This qualitative change in pattern caused by different settings of the parameter $\alpha$ requires the assignment of mutually exclusive sets of quantum numbers to the same set of eigenstates in different parameter regimes. The action quantum numbers $m, n$ are the trademark of quantum integrability. Their very existence accommodates level crossings between states of equal parity. The level sorting quantum number $k$, on the other hand, is applicable when level crossings between states of equal parity are prohibited. It is the trademark of quantum nonintegrability.

This conclusion brings us full circle to the thought experiment on invariant tori described in Sec. I. If we track an eigenstate along a closed path in the $(\Lambda, \alpha)$ plane, specifically a path that lies partly inside the integrable regime and partly outside, its individuality cannot be maintained through a unique and consistent assignment of quantum numbers. On a path that first leads a certain stretch through the integrable regime and then returns through the nonintegrable regime, the tagged eigenstate may undergo several crossings on the first leg of this path and will then, on the second leg, be unable to cross back to its initial position in the level sequence. Barring a minor caveat (see Appendix B) this conflict in the assignment of quantum numbers to eigenstates is a dependable detecting device for the demarcation of regimes of integrability and nonintegrability in quantum systems with few degrees of freedom.

\section{APPENDIX A: POINTS OF INTEGRABILITY}

Here we present additional results for the level spectrum, quantum invariants, and action operators of the spin-boson model (1) in the integrable regime, extending the findings of Secs. III-V to $\sigma>\frac{1}{2}$. The eigenvalue problem for $\sigma=1$ at integrability involves the solution of cubic equations. Here we list the $(\Lambda$-independent) eigenvectors and the associated energy eigenvalues for $\alpha=0$. We have $\left|\psi_{1,0}\right\rangle=|0,0\rangle,\left|\psi_{1,1}\right\rangle$ $=(|1,0\rangle+|0,1\rangle) / \sqrt{2},\left|\psi_{2,1}\right\rangle=(|1,0\rangle-|0,1\rangle) / \sqrt{2}$, with energies $E_{1,0}=-\hbar \omega, E_{1,1}=\sqrt{2} \Lambda, E_{2,1}=-\sqrt{2} \Lambda$, respectively, and for $n$ $\geq 2$ the results are

$$
\left|\psi_{1, n}\right\rangle=\sqrt{\frac{n-1}{4 n-2}}|0, n-2\rangle+\frac{1}{\sqrt{2}}|1, n-1\rangle+\sqrt{\frac{n}{4 n-2}}|2, n\rangle,
$$

$$
\begin{gathered}
\left|\psi_{2, n}\right\rangle=\sqrt{\frac{n}{2 n-1}}|0, n-2\rangle+\sqrt{\frac{n-1}{2 n-1}}|2, n\rangle, \quad(\mathrm{A} 1 \mathrm{~b}) \\
\left|\psi_{3, n}\right\rangle=\sqrt{\frac{n-1}{4 n-2}}|0, n-2\rangle-\frac{1}{\sqrt{2}}|1, n-1\rangle+\sqrt{\frac{n}{4 n-2}}|2, n\rangle,
\end{gathered}
$$

with energies

$$
\begin{gathered}
E_{1, n}=\hbar \omega(n-1)+\Lambda \sqrt{4 n-2}, \\
E_{2, n}=\hbar \omega(n-1), \\
E_{3, n}=\hbar \omega(n-1)-\Lambda \sqrt{4 n-2} .
\end{gathered}
$$

A simple analytic solution exists for arbitrary $\sigma$ in the asymptotic regime of large $n$. Consider the 
$(2 \sigma+1)$-dimensional invariant block of Eq. (1) at $\alpha=0$ formed by the basis vectors $|2 \sigma-m, n-m\rangle, m=0,1, \ldots, 2 \sigma$. It is tridiagonal with elements

$$
\begin{gathered}
\langle 2 \sigma-m, n-m|H| 2 \sigma-m, n-m\rangle=\hbar \omega(n-\sigma), \\
\langle 2 \sigma-m, n-m|H| 2 \sigma-m, n-m-1\rangle=\Lambda \sqrt{2 \sigma(n-m)} .
\end{gathered}
$$

For $n \gg \sigma$ we can write

$$
H=\hbar \omega(n-\sigma) \mathcal{E}+2 \Lambda \sqrt{n} S_{x}+\mathrm{O}\left(\frac{\sigma}{\sqrt{n}}\right),
$$

where $\mathcal{E}$ is the $(2 \sigma+1)$-dimensional unit matrix and $S_{x}$ is the irreducible representation of the spin operator with the same dimensionality. The asymptotic eigenvalues of this matrix are

$$
E_{m, n} \simeq \hbar \omega(n-\sigma)+2 \Lambda \sqrt{n}(\sigma-m)
$$

for $m=0, \ldots, 2 \sigma$. The corresponding analysis carried out for $\alpha=\pi / 2$ yields the matrix

$$
H=\hbar \omega(n-\sigma) \mathcal{E}+2 \hbar \omega S_{z}+2 \Lambda \sqrt{n} S_{x}+O\left(\frac{\sigma}{\sqrt{n}}\right)
$$

with asymptotic energy eigenvalues (for $m=0, \ldots, 2 \sigma$ )

$$
E_{m, n} \simeq \hbar \omega(n-\sigma)+2 \hbar \omega \sqrt{\lambda_{n}}(\sigma-m) .
$$

The quantum invariants (18) for $\sigma=1$ and $\alpha=0$ calculated from the eigenvectors (A1) are

$$
\langle A\rangle_{1, n}=-\langle A\rangle_{3, n}=\sqrt{n-1 / 2}, \quad\langle A\rangle_{2, n}=0 .
$$

Asymptotically for large $n$, we can evaluate the eigenvalues for arbitrary $\sigma$. The results for $\alpha=0$ read

$$
\langle A\rangle_{m, n}=(\sigma-m) \sqrt{n},
$$

and for $\alpha=\pi / 2$ we have

$$
\langle A\rangle_{m, n}=\frac{(\sigma-m) \sqrt{n}}{\sqrt{1+1 / n \lambda}} .
$$

The results of Sec. V for action operators are generalizable to arbitrary $\sigma$, albeit at the price of a higher and higher calculational effort. The case $\sigma=1, \alpha=0$ can still be presented compactly. The unitary transformation $U_{C}$ to be used in this case is now determined by the eigenvectors (A1) and yields

$$
\begin{gathered}
T_{z}=U_{C} S_{z} U_{C}^{-1}=P_{0}^{C}-G_{1}^{C} Q_{2}^{C}+\frac{1}{2} G_{2}^{C} P_{1}^{C}, \\
b^{\dagger} b=U_{C} a^{\dagger} a U_{C}^{-1}=\left(a^{\dagger} a+S_{z}+G_{1}^{C}\right) Q_{1}^{C}+\frac{1}{2} G_{2}^{C} P_{1}^{C},
\end{gathered}
$$

where

$$
\begin{aligned}
G_{1}^{C}= & \frac{S_{z}^{2}-S_{z}}{2 \sqrt{4 a^{\dagger} a-2}} a^{\dagger} S_{-}+\frac{1-S_{z}^{2}}{\sqrt{4 a^{\dagger} a+2}} a S_{+} \\
& +\frac{1-S_{z}^{2}}{\sqrt{4 a^{\dagger} a+2}} a^{\dagger} S_{-}+\frac{S_{z}+S_{z}^{2}}{\sqrt{4 a^{\dagger} a+6}} a^{\dagger} S_{+}, \\
G_{2}^{C} & =1+\frac{1-S_{z}^{2}}{\sqrt{2}} a S_{+}+\frac{S_{z}^{2}-S_{z}}{2 \sqrt{2}} a^{\dagger} S_{-},
\end{aligned}
$$

and $P_{0}^{C}=|0,0\rangle\left\langle 0,0\left|, P_{1}^{C}=\right| 1,0\right\rangle\langle 1,0|+| 0,1\rangle\langle 0,1|, Q_{2}^{C}=1-P_{0}^{C}$ $-P_{1}^{C}$. The transformed Hamiltonian becomes

$$
\begin{aligned}
U_{C} H U_{C}^{-1}= & \hbar \omega\left(b^{\dagger} b+T_{z}\right)+\frac{\Lambda}{\sqrt{2}}\left\{\left(3 T_{z}^{2}-S_{z}-2\right) P_{1}^{C}\right. \\
& \left.+\left[\left(T_{z}^{2}-T_{z}\right) \sqrt{2 b^{\dagger} b-1}-\left(T_{z}^{2}+T_{z}\right) \sqrt{2 b^{\dagger} b+3}\right] Q_{2}^{C}\right\} .
\end{aligned}
$$

Note that the transformations $U_{A}$ (for $\sigma=\frac{1}{2}$ ) and $U_{C}$ (for $\sigma$ $=1$ ), both pertaining to $\alpha=0$, are $\Lambda$ independent, whereas the transformation $U_{B}$, pertaining to $\alpha=\pi / 2$, is $\Lambda$ dependent.

\section{APPENDIX B: POINT OF HIGHER SYMMETRY}

Conflicts in the assignment of quantum numbers to eigenstates may arise for reasons unrelated to nonintegrability. In a study of a two-spin system [22] two such causes were identified: (i) the presence of points of higher symmetry inside the integrable regime; (ii) a multiple connectedness of the integrable regime in the parameter space. Both causes are readily identified as extraneous. In the context of the spinboson model (1) only the first cause comes into play.

In the following we describe one scenario where two eigenstates swap positions in the level spectrum when tracked along a closed path in parameter space, a path that does not leave the integrable regime. For this purpose we consider Eq. (1) with $\sigma=\frac{1}{2}$ in the extended parameter space $\left(\Lambda, \omega_{S}, \omega_{B}\right)$ at $\alpha=0$. The energy eigenvalues

$$
E_{ \pm}=\left(n+\frac{1}{2}\right) \hbar \omega_{B} \pm \frac{1}{2} \sqrt{4 \Lambda^{2}(n+1)+\left(\hbar \omega_{S}-\hbar \omega_{B}\right)^{2}}
$$

and the eigenvectors

$$
\begin{aligned}
& |+\rangle=\cos \phi|0, n\rangle+\sin \phi|1, n\rangle, \\
& |-\rangle=-\sin \phi|0, n\rangle+\cos \phi|1, n\rangle
\end{aligned}
$$

depend on the angular variable

$$
\phi=\arctan \frac{E_{+}-n \hbar \omega_{B}-\frac{1}{2} \hbar \omega_{S}}{\Lambda \sqrt{n+1}} .
$$

The point of higher symmetry is at $\Lambda=0, \omega_{B}=\omega_{S}$. Here the energy eigenvalues become doubly degenerate (for $\omega_{B}>0$ ). We consider the quantum invariant 


$$
\left\langle S_{z}\right\rangle_{ \pm}= \pm \frac{1}{2} \cos 2 \phi
$$

defined by expectation values in the eigenstates (B2). The loop in parameter space is parametrized as follows:

$$
\hbar \omega_{S}=\hbar \omega_{B}(1+\sin \beta),
$$

$$
\Lambda=\hbar \omega_{B}(1-\cos \beta),
$$

where $0 \leq \beta \leq 2 \pi$. It cuts through the point of higher symmetry at $\beta=0$. The crucial point is that one complete loop along this path advances the angle (B3) by $\Delta \phi=\pi / 2$, which interchanges the two states (B2) and does not bring both invariants (B1) and (B4) back to the same position. It takes two loops to return the states $| \pm\rangle$ to their original identity and the points $\left(E_{ \pm},\left\langle S_{z}\right\rangle_{ \pm}\right)$to their original position.
[1] M. Tabor, Chaos and Integrability in Nonlinear Dynamics (Wiley, New York, 1989).

[2] M. C. Gutzwiller, Chaos in Classical and Quantum Mechanics (Springer, New York, 1990).

[3] L. E. Reichl, The Transition to Chaos in Conservative Classical Systems: Quantum Manifestations (Springer, New York, 1992).

[4] R. M. Hilborn, Chaos and Nonlinear Dynamics (Oxford University Press, New York, 2000).

[5] Some aspects of this method were previously reported for a two-spin system (see Refs. [21-23]).

[6] M. B. Cibils, Y. Cuche, W. F. Wreszinski, J.-P. Amiet, and H. Beck, J. Phys. A 23, 545 (1990).

[7] M. B. Cibils, Y. Cuche, V. Marvulle, W. F. Wreszinski, J.-P. Amiet, and H. Beck, J. Phys. A 24, 1661 (1991).

[8] L. Müller, J. Stolze, H. Leschke, and P. Nagel, Phys. Rev. A 44, 1022 (1991).

[9] M. Cibils, Y. Cuche, and G. Müller, Z. Phys. B: Condens. Matter 97, 565 (1995).

[10] R. Graham, M. Höhnerbach, Z. Phys. B: Condens. Matter 57, 233 (1984).

[11] P. W. Milonni, J. R. Ackerhalt, and H. W. Galbraith, Phys. Rev. Lett. 50, 966 (1983).
[12] P. I. Belobrov, G. M. Zaslavskii, and G. Kh. Tartakovskii, Zh. Eksp. Teor. Fiz. 71, 1799 (1976) [Sov. Phys. JETP 44, 945 (1976)].

[13] The fundamental Poisson brackets for the physical variables are $\{p, x\}=1,\left\{S_{\alpha}, S_{\beta}\right\}=\Sigma_{\gamma} \epsilon_{\alpha \beta \gamma} S_{\gamma}$

[14] E. T. Jaynes and F. W. Cummings, Proc. IEEE 51, 89 (1963)

[15] The distinction between true crossings and barely avoided crossings (hard collisions) via numerical analysis becomes more and more difficult as the border to the integrable regime is approached.

[16] S. Weigert, Physica D 56, 107 (1992).

[17] S. Weigert and G. Müller, Chaos, Solitons Fractals 5, 1419 (1995).

[18] A. Peres, Phys. Rev. Lett. 53, 1711 (1984).

[19] N. Srivastava and G. Müller, Z. Phys. B: Condens. Matter 81, 137 (1990).

[20] N. Srivastava, C. Kaufman, G. Müller, R. Weber, and H. Thomas, Z. Phys. B: Condens. Matter 70, 251 (1988).

[21] V. V. Stepanov and G. Müller, Phys. Rev. E 58, 5720 (1998).

[22] V. V. Stepanov and G. Müller, Phys. Rev. E 62, 2008 (2000).

[23] V. V. Stepanov and G. Müller, Phys. Rev. E 63, 056202 (2001). 